DEPARTAMENTO IIF. ANATOMIA DESCRITIVA DOS ANIMAIS DOMÉSTICOS

DIRETOR: Prof. Dr. Orlando M. Paivo

\title{
COMPORTAMENTO DA ANSA SPIRALIS dO CólON EM BOVINOS AZEBUADOS *
}

(The arrangemene of the ansa spiralis of the zobu colon)

Orlando M. Paivo

Profossor Cotedrótico

Vicente Borelli Instrutor

No vasto e pouco explorad campo da anatomla dos bov 1 nos azebuados, elegemos por tema de investigação o comportamento da onso spirolis, uma das três porções do colon primum, homólogo do colon oscendens do homem (SUSSDORF, 1901; MRRTIN, 1912; ZISTZSCHMANN, 1925; KJUGER, 1929). Compreend1da entre a ansa proximalis $e$ a onsa distalis, a designação retrata-1he fielmente o arranjo, purquanto, a al ca espiral de inf́clo voltela centripetamente e a segulr centrlfuga mente, com us gyri centriperoles e gyri contrifugales a colocarem-se al ternados e equlidistantes, no mesmo plano. o arco de conversão dos giros centrlpetos aos centrífugos recebe o nome de flexuro controlis.

Em linhas gerals, esta descrição é endossada pela unan midade dos tratadistas que, entretanto, destoam a propósito do grau de enrolamento, omitem eventuals diferenças entre indivíduos dos dols sexos e não fazem qualquer alusão a antmals de raças Indianas

* Comunicado a XVII Conferencia Anual da Sociedade Paulista de Medicina Voterinória, realizada do 8 a 12 de setembro de 1962.

Trabalho efetuado sob os auspicios du Fundaço de Amparo a Pesquiso do Estoda de Söo Paulo. 
ou com sangue de zebu, pois, ostelam seus textos, invariavelmente, no Bos faurus.

O critério de contagem das voltas explica algumas discon dânclas. De fato, ou bem se considera o cólon convoluto dos mminan tes como alça en forma de $U$ alongado, enrod1unado sôbre si mesmo, com a base reproduzindo a flexura central e, então, face ao preguea mento do trato, o número de circuitos proxinals e distais é idênt1 co, ou bem, como querem SMITH $(1955,1957,1958,1959)$, SMITH e MEADOWS (1956), tomam-se por pontos de reparo secções defrontartes da primelra e última curvas, postas sôbre o prolongamento da Iinha que marca o I1mite dorsal do cltado segmento do intestino grosso, : - centro da espiral, disto decorrendo o registro de melo glou exeñ trico a mals (f18. 1).

Assim, culdando da seç̧ão intermediária do cólon, KIIMOV (1955), SCHWARZE e SCHRODER (1962) apontam uma e me1a voltas de entrada e outras tantas de safda; ELEENBERGER e BAUH (1932),

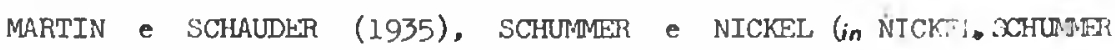
e SEIFERIE, 1960), AKAJEWSKI (1962) assinalam uma $c$ meia a duas centrlpetas e centrifugas; CARADONIA (in ZIMMERL, 1930) faz refe rencla a duas e (in BOSSI, CARADONNA, SPAMPANI, VARALDI e ZIMMERL, s.d.) duas e meia, em ambos os sentidos. Enfim, MARTIN, MONTANR, e BOURDELIE (1917), FAVILLI (1931) e, KitO (1960) mencionam dois a três lances proximais e três distais, enquanto LFSBRE (1922), ZIMMERL (1949), BRUNI e ZIMMERL (1951), DOBBERSTEIN E HOFFMANN (1963) indicam três.

Anal1sando o arranjo da onsa spirolis, em bovınos de raças européias, SMITH e MENDO's concluem que dos 735 exemplares sub metidos a exame, 711 ou $96,7 \%$ apresentam conflguração perfelta, 
repartindo-se os dados da seguinte manelra, cuanto a número de 81 ros concêntricos:

$$
\begin{aligned}
& \text { urr. - } 1 \text { vez }(0,14 \%) \text {, } \\
& \text { um e um quarto - } 1 \text { vez }(0,14 \%) \text {, } \\
& \text { um c melo - } 179 \text { vêzes }(24,35 \%) \text {, } \\
& \text { um e três quartos - } 7 \text { vêzes }(0,95 \%) \text {, } \\
& \text { do1s - } 520 \text { vêzes }(70,75 \%) \text {, } \\
& \text { dols e un quarto - I vez }(0,14 \%) \text {, } \\
& \text { do1s e me10 - } 2 \text { vêzes }(0,27 \%) \text {. }
\end{aligned}
$$

Nos carneiros, comenta $\circ$ A., O fndice global de regularidade do cólon convoluto chega a cêrca de $80 \%$ (em SMITH lêe-se 79,36\%), dependendo a diferença, talvez, da sua menor sloplicldade nẹ tes an1ma1s.

MATERIAI. E MÉTODO

Estudamos 1261 tratos 1ntestinals, separados de 1010 ma chos e 251 fêmear, de 1dades variáve1s, provindos de distintas zo nas de criação do Estado de São Paulo e abatidos no Frigorffico Armour da Cap1tal, onde, ao curso dos trabalhos de rotina, procede mos à verificação do sexo e ao assentamento da disposição das clreul tos, direta e nitidamente visfvels quando inspecionados pela esque da. Daquele total foram apartadas e mant1das em formol a $10 \%, 34$ peças portadoras de defeltos, restando-nos para aprec1ação, portan to, 980 do primeiro grupo e 247 do segundo.

Para marcação das voltas, cujas ocasionals superposições desprezamos, preferimos adotar a noma sugerida por SMITH (1955). 
K IES S I I. T A I) 0 S

Nos 1227 casos de espiralamento normal do cólon, somando $97,30^{\circ} \pm 0,46^{*}$ dos Indivfduos ut11izados, positivamos (quadro 1) que, mais amiúde, 1sto é, em 1034, fornecedores de $84,27 \% \pm 1,03$ das algas, existem duas curvas de entrada e duas e mela de salda. Wulpros 111, correspondentes a $9,05 \% \pm 0,82$ das observações no tam-se um e melo giros centrípetos e dols centrifugos. Apenas 82 vêzes, vale dizer, em $6,68 \% \pm 0,71$ dos antma1s, consignam-se duas e meia voltas proximais e três distais.

\section{Q U A D R O I}

Cólon esplral en bovino azebuados, segundo número de glros e sexos.

\begin{tabular}{|c|c|c|c|c|}
\hline Sexos & $1 \frac{1}{2}-2$ & $2-2 \frac{1}{2}$ & $2 \frac{1}{2}-3$ & Total \\
\hline M & 89 & 826 & 65 & 980 \\
\hline F & 22 & 208 & 17 & 247 \\
\hline Total & 111 & 1034 & 82 & 1227 \\
\hline
\end{tabular}

Interessando-nos conhecer se a distribulção dos cólons de vários feltios é diversa em ambos os sexos, calculamos a estatis tica $X^{2}$, achando-a 1gual a 0,025 , por conseguinte não significan te, quando comparada ao valor criftico para $5 \%$ e 3 graus de llberdade.

* desvio poriro 


\section{COMENTRRIOS E CONCIUSOES}

\section{Controntando os resultados obtidos com os de SMITH e}

MENDWS, atizentes a gado europeu, evidencia-se que, err têrmos de freqlência, os tipos de espirais estabelecidos nas duas pesquisas 으 ferecem 1dêntica ordenação, ou seja, aos de dols circuitos de entra da e dols e meio de safda, sucedem os de un e melo e dois, aparecen do afinal os de dois e meio e três. O emparelhamento das seriações envolve flagrantes disparidades percentuals, sobretudo no que tange a segunda e tercelra modalidades, quase igualmente representadas em nosso naterial, ao revés do aflançado pelos aludidos AA., para quem a últimf, constitul aspecto raro, face à penúltina, descoberta cêrca de uma vez soore cada quatro casos. Sob tal faceta, dir-se-1a algo malor a tendência a enrolamento da alça esplral hos uscuuauus, o que nos desobriga interpretemos tenham-na êstes nais longa, pois ainda recentemente demonstramos o contrár1o (PANVA e BORELLI, 1962). Corisoante SMITH e MENOWS, a mantfesta desproporção revelada pelo cotejo dos indices relativos a cólons convolutos 1 i vres de deformações, en bovinos (cêrca de 97,0, e ovinos (perto de 80,$0 ;)$, talvez dependa do número de giros concêntricos, varlá vel. respectivamente, de un a dols e meto e de dols a quatro. Váli da a preliminar e focando agora somente os grandes muninantes, o côm puto de tratos espirais regulares dos espécimes taurinos $\left(90,70^{\circ}\right)$, ivuco se destaca do relativo a zebuinos $(97,30 \%)$, acontecimento es ranhável, particularnente se ponderarmos que as parcelas de peças com, no minimo, duas voltas proximais e duas e mela distais elevam-se 
a $73,56 \%$ naqueles e a $90,95 \%^{\circ}$ nestes.

S U Y Y A A R Y

An analysis of the arrangement of the onsa spiralis of 1261 zebu cattle showed that $1227(97.30 \% \pm 0.46)$ had regular pat tems: $1034(84.27 \% \pm 1.03)$ were seen with two centripetal and two and a half centrifugal colls, $111(9.05 \% \pm 0.82)$ with one and a half centripetal and two centrifugal colls and $82(6.68, j \pm 0.71)$ with two and a half centripetal and three centrifugal co1ls. Sex did not influence the patterm of the ansa spiralis.

\section{REFERÊNCIAS BIBLIOGRÁFICAS}

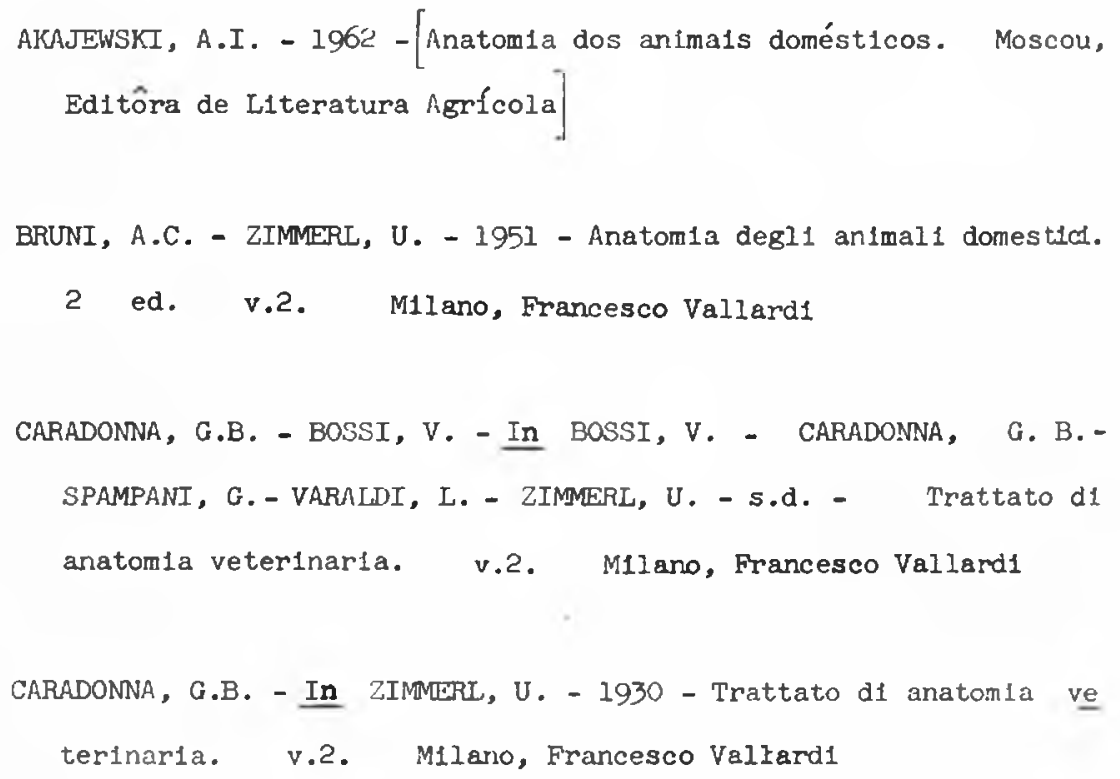


: OOBBERSTEIN, J. - IOFTMANN, G. - 1963 - Lehrbuch der vergletchenden finatomle der llaustlere. Bd. 2 Leipz1g, S. Hirzel

EIFNBRAGER, W. - ENUM, H. - 1932 - Hardbuch der very- erden Aratomfe der Haustiere. $\quad 17$ Auf. Berlin, Jull:is Spririger

FAVIIII, N. - 1931 - Nozlont comparate di Anatomia e Fistulogia dr gl1 animal1 rurall. Torío, Unicne T1pograflco :̈ditrice Torinese

KiTO, Y. - 1960 - finatomla comprativa dos antmals donésticos. 10 ed. v. 2. Tokyo, Yokendo \& C1a. Ltda

YIIHOV, A.F. - 1955-[ninatonia dos inima1s domésticos. T.I. Hoscou Editora de Literatura ABricola

ITUGER, W. - Ig29 - D1c vergle1chende Entwicklungsgeschichte im Dienste der LBsung der Homologislemungspoblems an den Darm - und Gekrbsabschnitten des Menschen und ełniger Haissäugetiero (Hund, Katze, Prerd, Schwein und wiederkluer ). Z. ges. frat., $20: 458-548$

LESBRE, F.X. - 1922 - Préc1s d' Anatom1e comparée àes animaux do mest1ques. T.1. Paris, J.B. Ballliere \& Fils

MRTIN, P. - I9!2 - Lehrbuch der Anatomie der Haustlere. a hidf. Bd. 4 Stuttgart, Sch1ckhard \& Eoner 
PARIN, P. - ICHAUDER, H. - 1935 - Lehrbuch der Anatomie der Haustierc. 3 fuf. Bd.3 T.2. Stuttgart, Schickhard $\therefore$ Ebner

MONTANE, I. - BOURLELLE, E. - 1317 - Anatomia regionale des animinex domestiquesi. T. 2. Parls, J.B. Ballilère \& Fils

PAIVA, O.M. - BORELII, V. - 1962 - Comprimento total do intestino em bovinos azebuados. Comuntcado à XVII Conferêncta Anual da da Sociedade Paulista de Medicina Veterinária, São Paulo.

SCHUMER, A. - ICKEL, R. - In NICKTLI, R. - SCHUMMER, A. SEIFELE, ז. - 1960 - Lehrbuch der Anatomle der Haustiere. Bd. 2. Berlin, Paul Parey

SCTWRRZL, E. - JCHRODER, L. - 1962 - Kompendium der veterindr anatomie. Bd. 2. Jena, Qustav Flscher

SISSON, S. - GROSSMAN, J.D. - 1945 - The anatomy of the domest1c animals. 3rd ed. Phlladelplhia, W.B., Saunders Co.

MMIT, R.N. - 1955 - The arrangement of the ansa spiralis of the sheep colon. J. Anat., 89 (2):246-249

NITH, R.R. \& MEADOWS, G.W. - 1956 - The arrangement of the ansa spiralis of the ox colon. J. Anat., $20(4): 523-526$ 
SMITH, R.N. - 1957 - The pattern of the ansa spiral1s of the sheep colon; (a) does it change (b) is it influenced by the sex of the sheep? Brit. vet. i., 113 (12):501-503

SMLTH, R.N. - 1958 - Irregular patterns of the ansa spirelis of the sheep colon. Brit. vet. J., $\underline{114}(8): 285-288$

SMITI, R.N. - 1959 - The arrangement of the anss splralts of the goat colon. Anat. Anz., $106(1 / 5): 101-103$

GUSSDORF, M. - 1901 - Homologien der Abschnitte des Dickdarms und dessen Gefässverte1lung. C1t.: EIIENBERGER, W. - BRIT, H. 1932

ZIFISCHMANN, 0. - 1955 - Der Darmkaral der stugetiere, efn verglelchend-anatomisches und entwlcklungsgeschichtiliches Proh lem. Anat. Anz., 60:155-172

ZIMMERL, U. - 1949 - Anatomia topogratica veterinaria. Milano, Francesco Vallardi. 
Rev. Fac. Med. Vet. S. Paulo-Vol. 7, fosc. 1, 1963-64

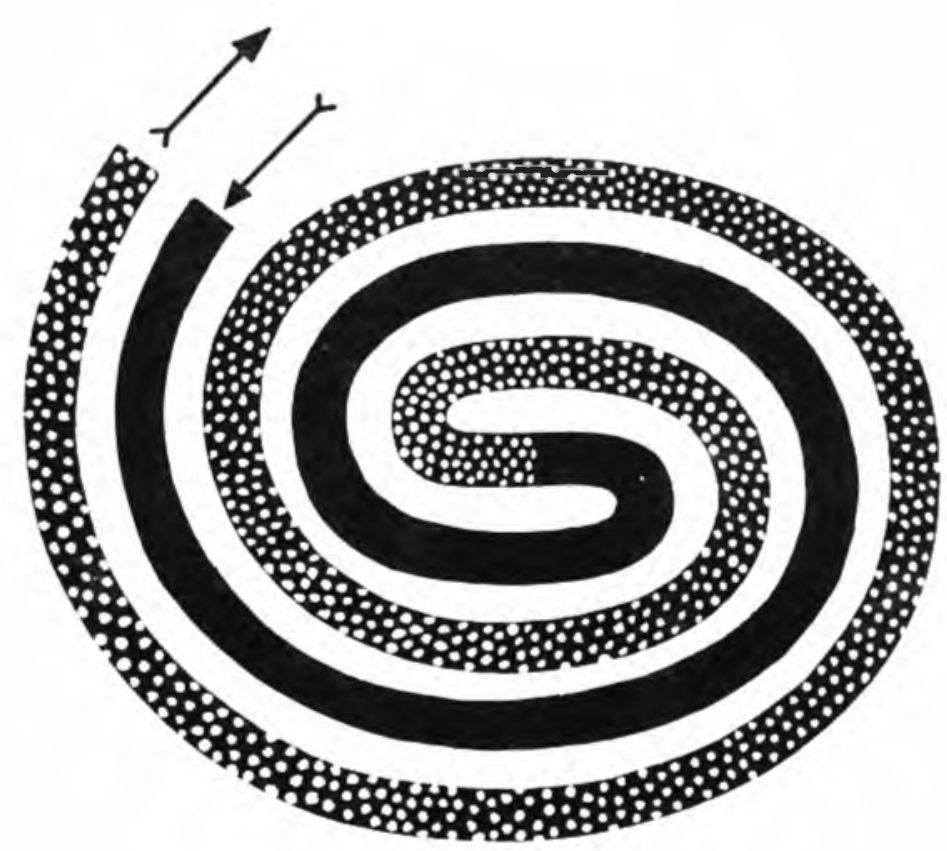

FIGURA 1 - Esquema da ansa spiralis do colon de bovino azebuado, com dois giros centrlpelos e dois e meio centrifugos, vistos pela esquerdo 